\title{
Detecting Misspelled Words in Turkish Text Using Syllable $n$-gram Frequencies
}

\author{
Rıfat Aşlıyan, Korhan Günel, and Tatyana Yakhno \\ Dokuz Eylül University, İzmir, Turkey
}

\begin{abstract}
In this study, we have designed and implemented a system which decides whether or not a word is misspelled in Turkish text. Firstly, three databases of syllable monogram, bigram and trigram frequencies are constructed using the syllables that are derived from five different Turkish corpora. Then, the system takes words in Turkish text as an input and computes the probability distribution of words using syllable monogram, bigram and trigram frequencies from the databases. If the probability distribution of a word is zero, it is decided that this word is misspelled. For testing the system, we have constructed two text databases with the same words. One text database has 685 misspelled words. The other has 685 correctly spelled words. The words from these text databases are taken as inputs for the system. The system produces two results for each word: "Correctly spelled word" or "Misspelled word". The system that is designed with monogram and bigram frequencies has $86 \%$ success rate for the misspelled words and has $88 \%$ success rate for the correctly spelled words. According to the system designed with bigram and trigram frequencies, there is $97 \%$ success rate for the misspelled words and there is $98 \%$ success rate for the correctly spelled words.
\end{abstract}

\section{Introduction}

To detect misspelled words in a text is an old problem. Today, most of word processors include some sort of misspelled word detection. Misspelled word detection is worthy in the area of cryptology, data compression, speech synthesis, speech recognition and optical character recognition [1, 2, 3] 4]. The traditional way of detecting misspelled words is to use a word list, usually also containing some grammatical information, and to look up every word in the word list [6] from dictionary.

The main disadvantage of this approach is that if the dictionary is not large enough, the algorithm will report some of correct words as misspelled, because they are not included in the dictionary. For most natural languages the size of dictionary needed is too large to fit in the working memory of an ordinary computer. In Turkish this is a big problem, because Turkish is an agglutinative language and too many new words can be constructed by adding suffixes.

To overcome this difficulties we have proposed a new approach for detecting misspelled words in Turkish text. We have used Turkish syllable $n$-gram 
frequencies which are generated from several Turkish corpora [5] [7]. From the corpora we have extracted syllable monogram, bigram and trigram frequencies using TASA (Turkish Automatic Spelling Algorithm) 8]. We have used these $n$-gram frequencies for calculating a word probability distribution. After that the system has decided whether a word is misspelled or not. In this approach we don't need word list. We have only Turkish syllables and their monogram, bigram and trigram frequencies.

The paper is organized as follows. In Section 2, we described the system architecture. We explained how syllable $n$-gram frequencies and word probability distribution are computed. We discussed the empirical results of the system in Section 3. Future directions and coclusions are described in Section 4.

\section{System Architecture}

The system consists of three main components. First component is preprocessing which cleans a text. Second component is TASA, and third component is calculating probability distribution of words. As shown in Figure 1, the system takes words in Turkish text as input and gives the result for each word as "Misspelled Word" or "Correctly Spelled Word".

In preprocessing component of the system, punctuation marks are cleaned. All letters in the text are converted to lower case. Blank characters between two successive words are limited with only one blank character.

In second component, TASA [8] takes the Turkish clean text as an input and gives the Turkish syllabified text. The system divides words into syllables putting the dash character between two syllables. For example, the word "kitaplik" in Turkish text is converted into the syllabified word "ki-tap-lık" in Turkish syllabified text.

In third component, the probability distribution is calculated for each syllabified word. The system uses syllable monogram, bigram and trigram frequencies to find this probability distribution. How these $n$-gram frequencies are computed is explained in detail in the following Section 2.1.

\subsection{Calculation of Syllable $n$-gram Frequencies}

We have used the Turkish corpora 5] 7] which includes 304178 Turkish words and the corpora is preprocessed as seen in Figure 1. The system TASA syllabifies all Turkish words in the corpora. We have constructed Turkish syllable corpora from the Turkish word corpora. Turkish syllable corpora contains 900342 Turkish syllables. As shown in Table 1, Table 2 and Table 3. Turkish syllable monogram, bigram and trigram frequencies are calculated. For example, the frequency of the syllable monogram "la" is 21322. In Table 2 and Table 3 "blank" represents only one blank character. We accepted blank character as syllable for the system. Table 2 shows the frequencies of some Turkish syllable bigram. 


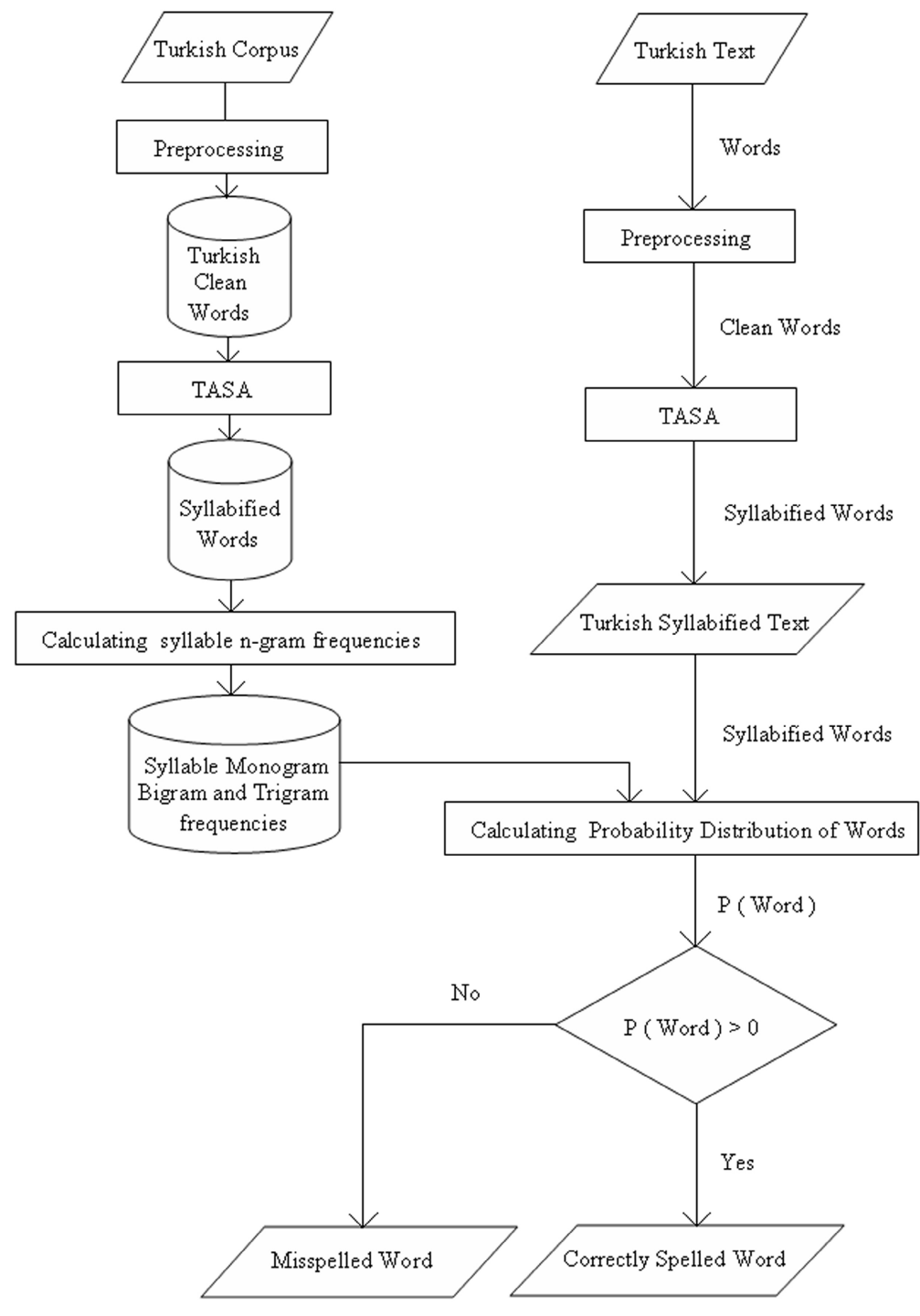

Fig. 1. System architecture

\subsection{Calculation of the Probability Distribution of Words}

An $n$-gram is a sub-sequence of $n$ items from a given sequence. $n$-grams are used in various areas of statistical natural language processing and genetic sequence analysis. The items in question can be letters, syllables, words according to the application. 
Table 1. Monogram statistics for Turkish corpus

\begin{tabular}{|c|c|c|c|c|c|c|}
\hline \multicolumn{2}{|c|}{ Monogram Frequency } & \multicolumn{2}{|c|}{$\% \mid$ Monogram Frequency } & \multicolumn{3}{|c|}{$\% \mid$ Monogram Frequency } \\
\hline la & 21322 & 2.37 |ra & 11611 & $1.29 \mid \mathrm{ec}$ & 5909 & 0.66 \\
\hline ma & 17704 & $1.97 \mathrm{da}$ & 10930 & 1.21 rih & 5186 & 0.58 \\
\hline li & 15124 & 1.68 ve & 10570 & 1.17 mak & 3654 & 0.41 \\
\hline $\mathrm{a}$ & 13439 & 1.49 ri & 9618 & $1.07 \mathrm{di}$ & 3022 & 0.34 \\
\hline ta & 13372 & $1.48 \mid \mathrm{r} 1$ & 9105 & $1.01 \mathrm{ne}$ & 2788 & 0.31 \\
\hline i & 12827 & $1.42 \mathrm{me}$ & 8776 & 0.97 si & 2741 & 0.30 \\
\hline de & 11699 & $1.30 \mathrm{e}$ & 7312 & 0.81 mek & 2718 & 0.30 \\
\hline
\end{tabular}

Table 2. Bigram statistics for Turkish corpus

\begin{tabular}{|c|c|c|c|c|c|c|}
\hline |Bigram & Frequency & $\% \mid B i g r a m$ & Frequency & $\%$ Bigram & Frequency & $\%$ \\
\hline & 12880 & 1.43|blank & & 0.88 rih li & & 0.55 \\
\hline & & $1.24 \mathrm{da}$ & & 0.82 bla & & 0.52 \\
\hline nk ve & 9 & 1.09 ec blank & & $0.65 \mathrm{ll} \mathrm{bl}$ & & 0.51 \\
\hline & & 1.07 la rı & & $.63 \mathrm{rl} \mathrm{bl}$ & & 0.46 \\
\hline & 9410 & 1.04 le ri & 5551 & 0.62 ma blank & 3703 & 0.41 \\
\hline lank & & 0.98 ta rih & 5186 & $0.58 \mid \mathrm{ma} \mathrm{s} 1$ & & 0.41 \\
\hline blank ta & 8296 & 0.92 le blank & 5109 & 0.57 mak blank & 3558 & 0.39 \\
\hline
\end{tabular}

Table 3. Trigram statistics for Turkish corpus

\begin{tabular}{|lrr|lrr|}
\hline Trigram & Frequency & $\%$ & Trigram & Frequency & $\%$ \\
\hline blank ve blank & 8566 & 0.05 & e ec blank & 3227 & 0.02 \\
blank ta rih & 5238 & 0.03 & blank i le & 3037 & 0.02 \\
ta rih li & 4944 & 0.03 & blank bir blank & 3000 & 0.02 \\
rih li blank & 4944 & 0.03 & i le blank & 2997 & 0.02 \\
blank i liş & 3437 & 0.02 & la r r blank & 2979 & 0.02 \\
i liş kin & 3282 & 0.02 & le ri blank & 2905 & 0.02 \\
blank e ec & 3228 & 0.02 & blank kon sey & 2896 & 0.02 \\
kon sey blank & 2895 & 0.02 & blank i çin & 2750 & 0.01 \\
blank ko mis & 2606 & 0.01 & blank ka ra & 2578 & 0.01 \\
ko mis yon & 2515 & 0.01 & mis yon blank & 2514 & 0.01 \\
\hline
\end{tabular}

An $n$-gram of size 1 is a "monogram"; size 2 is a "bigram"; size 3 is a "trigram"; and size 4 or more is simply called an " $n$-gram" or " $(n-1)$-order Markov model" 9].

An $n$-gram model predicts $x_{i}$ based on $x_{i-1}, x_{i-2}, x_{i-3}, \ldots, x_{i-n}$. When used for language modeling independence assumptions are made so that each word depends only on the last $n$ words. This Markov model is used as an approxima- 
tion of the true underlying language. This assumption is important because it massively simplifies the problem of learning the language model from data.

Suppose that a word $W$ in Turkish syllabified text consists of the syllable sequence $s_{1}, s_{2}, s_{3}, \ldots, s_{t}$. This word has $t$ syllables. To obtain the $n$-gram probability distribution [10] of the word $W$, we have used formula (11).

$$
P(W)=P\left(s_{1}, s_{2}, s_{3}, \ldots, s_{t}\right)=\prod_{j-1}^{t} P\left(s_{i} \mid s_{i-n+1}, s_{i-n+2}, \ldots, s_{i-2}, s_{i-1}\right)
$$

In $n$-gram model, the parameter $P\left(s_{i} \mid s_{i-n+1}, s_{i-n+2}, \ldots, s_{i-2}, s_{i-1}\right)$ in the formula (1) can be estimated with Maximum Likelihood Estimation (MLE) 8] technique as shown in formula (2).

$$
P\left(s_{i} \mid s_{i-n+1}, s_{i-n+2}, \ldots, s_{i-2}, s_{i-1}\right) \approx \frac{C\left(s_{i-n+1}, s_{i-n+2}, \ldots, s_{i-1}, s_{i}\right)}{C\left(s_{i-n+1}, s_{i-n+2}, \ldots, s_{i-1}\right)}
$$

So, we conclude as formula (3).

$$
P(W)=P\left(s_{1}, s_{2}, s_{3}, \ldots, s_{t}\right) \approx \prod_{i=1}^{t} \frac{C\left(s_{i-n+1}, s_{i-n+2}, \ldots, s_{i-1}, s_{i}\right)}{C\left(s_{i-n+1}, s_{i-n+2}, \ldots, s_{i-1}\right)}
$$

In formula (2) and (3), $C\left(s_{i-n+1}, s_{i-n+2}, \ldots, s_{i-1}, s_{i}\right)$ is the frequency of the syllable sequence $s_{i-n+1}, s_{i-n+2}, \ldots, s_{i-1}, s_{i}$. Furthermore, $C\left(s_{i-n+1}, s_{i-n+2}\right.$, $\left.\ldots, s_{i-1}\right)$ is the frequency of the syllable sequence $s_{i-n+1}, s_{i-n+2}, \ldots, s_{i-1}$. The frequencies of these syllable sequences can be calculated from the Turkish corpora [5].

For $\operatorname{bigram}(n=2)$ and $\operatorname{trigram}(n=3)$ models, probability distribution $P(W)$ can be computed as shown in formula (4) and (5) respectively.

$$
\begin{gathered}
P(W)=P\left(s_{1}, s_{2}, s_{3}, \ldots, s_{t}\right)=\prod_{i=1}^{t} P\left(s_{i} \mid s_{i-1}\right) \approx \prod_{i=1}^{t} \frac{C\left(s_{i-1}, s_{i}\right)}{C\left(s_{i-1}\right)} \\
P(W)=P\left(s_{1}, s_{2}, s_{3}, \ldots, s_{t}\right)=\prod_{i=1}^{t} P\left(s_{i} \mid s_{i-2}, s_{i-1}\right) \approx \prod_{i=1}^{t} \frac{C\left(s_{i-2}, s_{i-1}, s_{i}\right)}{C\left(s_{i-2}, s_{i-1}\right)}
\end{gathered}
$$

For example, according to bigram model we can calculate the probability distribution of a word in Turkish text. Assume that we have a text which includes some words as shown in (6). This text is converted to syllabified text as (7). Syllables are delimited with dash character.

$$
\text { "... bu gün okulda şenlik var..." }
$$

$$
\text { “.. bu gün o-kul-da şen-lik var..." }
$$


Assume that the word $W=$ "okulda" in (6) is taken for computing its probability distribution and $W$ can be written as the syllable sequence $W=s_{1}, s_{2}, s_{3}=$ "o", "kul", "da" as shown in (7). Here, $s_{1}=$ "o", $s_{2}=$ "kul", $s_{3}=$ "da". We accepted blank character as a syllable. We call this syllable as $\lambda$. So, assume that syllable monogram frequencies are $C($ " $\lambda$ " $)=0.003, C($ "o" $)=0.002, C$ ("kul") $=0.004$ and syllable bigram frequencies are $C(" \lambda$ ", "o" $)=0.0001, C($ "o", "kul" $)=0.0002$, $C$ ("kul", "da") $=0.0003$. We have calculated $P$ ( "okulda") using bigram model. We have found that the probability distribution of the word "okulda" is 0.0002475 as shown in (8).

$$
\begin{gathered}
P(W)=P(\text { "okulda" })=P\left(s_{1}, s_{2}, s_{3}\right)=P(\text { "o", "kul", "da" }) \\
=\prod_{i=1}^{3} P\left(s_{i} \mid s_{i-1}\right) \approx \prod_{i=1}^{3} \frac{C\left(s_{i-1} \mid s_{i}\right)}{C\left(s_{i-1}\right)} \\
=\left(\frac{C(" \lambda ",, " \mathrm{o} "}{C(" \lambda ")}\right)\left(\frac{C(\text { "o", "kul" }}{C(" \mathrm{o} ")}\right)\left(\frac{C(\text { "kul", "da" }}{C(\text { "kul" })}\right)
\end{gathered}
$$

\section{Testing the System}

We have designed and implemented two systems to detect misspelled words in Turkish text. One uses monogram and bigram frequencies. The size of monogram database is 41 kilobytes and our monogram consists of 4141 different syllables. The size of bigram and trigram databases are 570 and 2858 kilobytes respectively. While the bigram database includes 46684 syllable pairs, the trigram database consists of 183529 ternary syllables. The other uses bigram and trigram frequencies. We have tested these two systems. To test the systems, we have two Turkish texts. One is correctly spelled text which includes 685 correctly spelled words. The other is misspelled text which has 685 misspelled words. These two texts have same words. Namely, misspelled words are generated with putting errors on the correctly spelled words. These error types are substitution, deletion, insertion, transposition and split word errors. The systems takes correctly spelled and misspelled texts as input and gives the results for each word as "correctly spelled word" or "misspelled word". As it is shown in Figure 1, probability distributions are calculated for each word. If the probability distribution of a word is equal to zero, system decides that the word is misspelled. If it is greater than zero, system decides that the word is correctly spelled.

The system works with Intel-based NT, Windows 2000, XP, Windows 2003 Server systems with 512MB RAM and it has been developed using Borland $\mathrm{C}++$ Builder Professional.

We have first tested the system on the correctly spelled text using monogram and bigram frequencies. The system determines 602 correctly spelled words from the correctly spelled text, so the words are correctly recognized with $88 \%$ success rate. Also, 589 misspelled words within the misspelled text are decided successfully by the system. Namely, the system which is tested on the misspelled text correctly recognized the words with $86 \%$ success rate. 
Finally we have tested the system on the correctly spelled text using bigram and trigram frequencies. The system determines 671 of 685 correctly spelled words from the correctly spelled text. The success rate on correctly recognition of the words is $98 \%$. Furthermore, 664 of 685 misspelled words within the misspelled text are decided successfully by the system. Thus, the system which is tested on the misspelled text correctly recognized the words with $97 \%$ success rate. Our system performance is competitive with similar systems. The system checks the approximately 75.000 words per second.

\section{Conclusion}

In this study, we have presented two systems to detect misspelled words on Turkish text. The system which uses syllable monogram and bigram frequencies is quite successful to find misspelled words. The system's success rate is $86 \%$ to detect misspelled words on Turkish text. But, we had better success rate when we develop the system that uses syllable bigram and trigram frequencies. This system reached $97 \%$ success rate to detect misspelled words.

For future directions, we plan to develop a system which uses syllable trigram and 4-gram frequencies to find misspelled words. Syllable $n$-gram frequencies are very important for our system. Therefore, we plan to extract new syllable $n$-gram frequencies from different Turkish corpora.

\section{References}

1. Barari, L., QasemiZadeh, B.: CloniZER spell checker adaptive language independent spell checker. In: AIML 2005 Conference CICC, Cairo, Egypt, pp. 19-21 (2005)

2. Tong, X., Evans, D.A.: A statistical approach to automatic OCR error correction in context. In: Proceedings of the Fourth Workshop on Very Large Corpora, Copenhagen. Denmark, pp. 88-100 (1996)

3. Kang, S.S., Woo, C.W.: Automatic segmentation of words using syllable bigram statistics. In: Proceedings of the Sixth Natural Language Processing Pacific Rim Symposium, Tokyo. Japan, November 27-30, pp. 729-732 (2001)

4. Deorowicz, S., Ciura, M.G.: Correcting spelling errors by modelling their causes. International Journal of Applied Mathematics and Computer Science 15(2), 275285 (2005)

5. Dalkilic, G., Cebi, Y.: Creating a Turkish corpus and determining word length. DEU Muhendislik Fakultesi Fen ve Muhendislik Dergisi 5(1), 1-7 (2003)

6. Kukich, K.: Techniques for automatically correcting words in text. ACM Computing Surveys 24(4), 377-439 (1992)

7. Kurumu, T.D.: Imla kilavuzu. Ankara (2001)

8. Asliyan, R., Günel K.: Design and implementation for extracting Turkish syllables and analyzing Turkish syllables. In: International Symposium on Innovations in Intelligent Systems and Applications. INISTA (2005)

9. Zhuang, L., Bao, T., Zhu, X., Wang, C., Naoi, S.: A chinese OCR spelling check appoarch based on statistical language models. IEEE International Conference on Systems, Man and Cybernetics, 4727-4732 (2004)

10. Jurafsky, D., Martin, J.H.: Speech and language processing. Prentice-Hall Press, Englewood Cliffs (2000) 